\section{Uso de suplementos vitamínicos e/ou minerais por crianças menores de seis meses no interior do estado de Pernambuco}

\section{Use of vitamin and/or mineral supplies by infants under six months of age in the interior of Pernambuco State}

Maria de Fátima Gallindo de Melo 1

Leonor Maria Pacheco Santos 2

Pedro Israel Cabral de Lira 3

1,3 Departamento de Nutrição. Centro de Ciências da Saúde. Universidade Federal de Pernambuco. Av. Moraes Rego s. n. Cidade Universitária. Recife, PE, Brasil. CEP 50.670-901 E-mail: fatimagallindo@hotmail.com 2 Organização Pan Americana da Saúde. Organização Mundkal da Saúde. Brasília, DF, Brasil

\section{Abstract}

Objectives: to describe the use of vitamin and or minerals supplements in children from birth to six months of age, as well as to assess the association between supplements use and social, economic, biological features, nutrition standards and healthcare.

Methods: the study comprised a sub-sample of 399 children in a cohort study performed in the interior of Pernambuco.

Results: the proportion of children receiving vitamin and/or minerals supplements was of $18.8 \%$. The use of these supplements increased with the child's age, OR=4.38 with 17 weeks and $O R=9.82$ with 26 weeks of age when compared with four weeks old infants. The increased use of supplements was determined in children of low income families and among the ones not sharing the home with other children under five years old. Twenty five year old or older mothers used more supplements than younger mothers. The average time for breastfeeding was associated to vitamins and/or minerals supplements use, being shorter among children using these supplements.

Conclusions: the use of supplements as a means for vitamins and/or minerals offer in babies whose nutritional needs could be met through exclusive breastfeeding, was determined.

Key words Dietary supplements, Child health (Public Health), Vitamins, Dietary minerals

\section{Resumo}

Objetivos: descrever o uso de suplementos vitamínicos elou minerais em crianças, do nascimento até o sexto mês de vida, bem como avaliar a associação entre o uso dos suplementos e características socioeconômicas, biológicas, padrões alimentares e de assistência à saúde.

Métodos: o estudo compreendeu uma sub-amostra de 399 crianças que pertenciam a um estudo coorte, realizado no interior de Pernambuco.

Resultados: a proporção de crianças que recebeu vitaminas elou minerais foi de 18,8\%. O uso desses suplementos aumentava com a idade da criança, OR=4,38 com 17 semanas e OR=9,82 com 26 semanas de vida, quando comparadas com as crianças na idade de quatro semanas. $O$ maior uso de suplementos foi observado naquelas crianças de melhor renda e entre aquelas que não compartilhavam o domicílio com outras menores de cinco anos. As mães com idade igual ou superior a 25 anos recorreram mais à suplementação para seus filhos do que aquelas mais jovens. A duração mediana do aleitamento materno esteve associada à suplementação de vitaminas elou minerais, sendo menor entre as crianças que utilizaram esses medicamentos.

Conclusões: verificou-se a utilização do recurso medicamentoso como via de ingestão de vitaminas elou minerais em lactentes cujas necessidades nutricionais poderiam ser atendidas através do aleitamento materno exclusivo.

Palavras-chave Suplementos dietéticos, Saúde infantil (Saúde Pública), Vitaminas, Minerais na dieta 


\section{Introdução}

A importância assumida pelos medicamentos na prática da assistência à saúde está intimamente relacionada ao raciocínio mecanicista que norteia o modelo biomédico, assim como às transformações operadas nas formas de produzir e consumir remédios. ${ }^{1}$

O Brasil vem se destacando como um dos maiores consumidores de medicamentos. No início da década de 90 encontrava-se entre os sete maiores mercados do mundo, ${ }^{1}$ passando em 1998 a ser considerado o quinto maior produtor mundial de medicamentos. ${ }^{2}$ Em se tratando do varejo farmacêutico, predomina a lógica de mercado, gerando-se uma tensão entre a necessidade sanitária dos medicamentos e a necessidade constante de expansão do mercado. A exigência de prescrição médica não é observada, predominando os procedimentos de incremento da venda de produtos mais caros e desnecessários.2,3

Dentro desse contexto, os suplementos vitamínicos têm sido bastante explorados pela indústria farmacêutica, na qualidade de produtos promotores da saúde. Para as vitaminas comercializadas sob a forma de medicamentos, são atribuídas propriedades cuja representação simbólica faz com que os nutrientes contidos nesses produtos passem a ser vistos como mais eficazes do que aqueles mesmos elementos disponíveis nos alimentos. 1 Tratando sobre os problemas carenciais dos países subdesenvolvidos, Chetley alerta para o fato de que a ampla difusão dos produtos vitamínicos contribui para "...medicalizar o indivíduo deixando intocadas as causas sociais e econômicas da desnutrição". (1994: 249). 4

Por outro lado, a utilização racional dos medicamentos pode promover o alívio e a cura de várias enfermidades. A questão das deficiências de micronutrientes e seu efeito no retardo do crescimento, na morbidade e na mortalidade infantil é um importante problema de saúde pública, expressandose pelo impacto substancial que representam no nível de saúde e indicadores da qualidade de vida das populações. 5 A utilização de preparações vitamínicas como suplementos dietéticos ou em doses terapêuticas pode ser recomendada, ressaltando-se a necessidade da prescrição médica no uso de doses superiores às necessidades nutricionais, casos nos quais as vitaminas passam a ter ação farmacológica cujos riscos devem ser avaliados. 6,7

No caso de crianças menores de seis meses de idade, a suplementação de algumas carências de micronutrientes como ferro e vitamina A tem sido motivo de controvérsias. ${ }^{8-10}$ Em se tratando do ferro, os riscos e benefícios da suplementação em diversas populações não têm sido bem documentados, havendo também controvérsias sobre o efeito da suplementação no crescimento e morbidade. ${ }^{8}$

Uma outra forma de abordar a questão das deficiências de micronutrientes é apresentada em um estudo realizado entre crianças mexicanas, cujos autores discutiram a questão da deficiência múltipla de micronutrientes, comum em populações pobres, chamando a atenção para o fato de que é possível que mais de um micronutriente seja responsável pela limitação do crescimento. Nesse estudo, abrangendo a faixa etária de 8 a 12 meses de idade, os autores concluíram sobre um maior efeito da suplementação no crescimento das crianças mais jovens. Nessas, as necessidades de micronutrientes são maiores, devido à maior velocidade do crescimento, como também ao fato de que suas dietas são mais restritas quando comparadas com as de crianças mais velhas. 11

Alguns estudos sobre o padrão de utilização de medicamentos apontam para um uso abusivo em crianças, estando os suplementos vitamínicos entre os produtos mais empregados.12-18 No entanto, são escassas as pesquisas com ênfase específica para o uso de medicamentos nos primeiros seis meses de vida, época especialmente importante para a saúde infantil. Em revisão bibliográfica nos últimos 10 anos encontrou-se apenas um estudo que examinou o consumo de medicamentos em crianças no primeiro trimestre de vida16 e um outro que investigou a suplementação vitamínica em crianças nos seis primeiros meses de vida e aos três anos de idade. 19

Dessa forma, o objetivo do presente estudo é descrever o uso de suplementos vitamínicos e/ou minerais em crianças, do nascimento até o sexto mês de vida, bem como avaliar a associação entre o uso dos suplementos e características socioeconômicas, biológicas, padrões alimentares e de assistência à saúde.

\section{Métodos}

A presente investigação baseou-se em um banco de dados gerado por um estudo de coorte, realizado na Zona da Mata Meridional de Pernambuco, Brasil, nos municípios de Palmares, Ribeirão, Catende, Água Preta e Joaquim Nabuco, nos anos de 1993 a 1994. Entre esses municípios, Palmares é o maior deles, possuindo uma população de 60.000 habitantes; e, de acordo com o Censo Demográfico de 1991, $81 \%$ das famílias recebiam menos de dois salários mínimos. 20 
A referida coorte era constituída por 468 crianças nascidas a termo, de baixo peso (1500g a $2499 \mathrm{~g}$ ) e de peso adequado ao nascer (3000g a $3499 \mathrm{~g}$ ), acompanhadas nos primeiros seis meses de vida. Essa amostra recrutada correspondeu a aproximadamente $20 \%$ de um total de 2200 partos ocorridos nas maternidades durante o período estudado. Dados rotineiramente coletados em 1993 demonstraram que a prevalência de baixo peso ao nascer foi de $9,6 \% .20$

Para a coleta de dados sobre o consumo de medicamentos foi realizado um inquérito de consumo nos momentos em que as crianças completavam 4, 8, 17 e 26 semanas de vida, para verificação da ingestão alimentar e da utilização de produtos farmacêuticos (vitaminas, minerais, pomadas e outros medicamentos). Foi considerado uso de medicamento somente a informação afirmativa ou negativa em relação à utilização, independente da dose e da frequiência.20

Foram utilizados neste estudo os dados das 399 crianças que permaneceram na coorte até o final do acompanhamento (26 semanas), das quais 164 nasceram com baixo peso (média: $2,341 \mathrm{~g}, \mathrm{DP}=0,154$ ) e 235 com peso adequado (média: 3,206g, DP=0,143).

As variáveis estudadas na presente investigação foram as seguintes: variável dependente - uso de suplementos vitamínicos e/ou minerais; variáveis independentes - idade da criança, renda familiar mensal em salários mínimos, peso ao nascer, sexo da criança, número de pessoas no domicílio, escolaridade da mãe, condições de habitação e saneamento (tipo de parede e de piso, água, lixo e destino dos dejetos), posse de bens de consumo (rádio, televisão, geladeira e fogão), idade da mãe, número de crianças menores de cinco anos no domicílio, número de consultas pré-natais, consultas médicas e hospitalizações da criança durante todo o acompanhamento, duração do aleitamento materno e estado nutricional da criança (escores $z$ para os indicadores peso/idade e altura/idade), sendo utilizado como referência de normalidade o padrão do National Center for Health Statístics (NCHS). ${ }^{21}$ Para verificar a associação entre o estado nutricional e o uso de suplementos, foi escolhida a idade de seis meses, uma vez que o uso de suplementos foi maior nessa idade.

Para o processamento e análise estatística dos dados, todas as informações foram digitadas e analisadas em Epi-info 6.04b. Os testes estatísticos utilizados foram o qui-quadrado, para associação com as variáveis categóricas, e o teste nãoparamétrico Mann-Whitney-U, para a comparação com a duração da mediana do aleitamento materno em dias, adotando-se o nível de significância de 5\%.

Para a estimativa do uso de suplementos vitamínicos de acordo com a idade tomou-se como referência as crianças na quarta semana de vida, estimando-se o Odds ratio (OR) e respectivos intervalos de confiança para as idades de 8, 17 e 26 semanas, tendo sido adotado também um nível de significância de $5 \%$.

\section{Resultados}

Das 399 crianças estudadas, 75 (18,8\%) usaram algum suplemento vitamínico e/ou mineral, totalizando 88 episódios de uso medicamentoso ao longo do acompanhamento, em decorrência do uso repetido por algumas crianças nas diferentes idades. $\mathrm{O}$ uso de suplementos vitamínicos e/ou minerais nas faixas etárias de 17 e 26 semanas foi significativamente maior $(p<0,001)$, com valores de OR de 4,38 (IC95\%: 1,7-12,0) e 9,82 (IC95\%: 4,0-25,7), respectivamente, quando comparado com o das crianças na idade de quatro semanas (Tabela 1).

Os grupos farmacológicos mais consumidos (Tabela 2) foram os recalcificantes $(56,8 \%)$, os polivitamínicos $(18,0 \%)$ e os antianêmicos $(5,7 \%)$.

A análise da situação nutricional demonstra que, ao completar vinte e seis semanas de vida, 49

\section{Tabela 1}

Uso de suplementos vitamínicos e/ou minerais segundo a idade das crianças. Zona da Mata Meridional de Pernambuco, 1993 a 1994

\begin{tabular}{lrrrrrrrr}
\hline Idade (semanas) & \multicolumn{3}{c}{ Utilização de suplementos } & Total & OR* (IC95\%) & P \\
\hline & $\mathrm{n}$ & $\%$ & $\mathrm{n}$ & $\%$ & & & \\
\hline 4 & 6 & 1,5 & 393 & 98,5 & 399 & 1,00 & \\
8 & 5 & 1,3 & 394 & 98,7 & 399 & $0,83(0,2-3,1)$ & 1,00 \\
17 & 25 & 6,3 & 374 & 93,7 & 399 & $4,38(1,7-12,0)$ & $<0,001$ \\
26 & 52 & 13,0 & 347 & 87,0 & 399 & $9,82(4,0-25,7)$ & $<0,001$ \\
\hline
\end{tabular}

* Odds ratio e Intervalo de Confiança de $95 \%$. 
Tabela 2

Distribuição percentual dos grupos farmacológicos consumidos. Zona da Mata Meridional de Pernambuco, 1993 e 1994

\begin{tabular}{lrr}
\hline Grupos farmacológicos & N & $\%$ \\
\hline Recalcificantes & 50 & 56,8 \\
Polivitamínicos & 16 & 18,2 \\
Antianêmicos & 5 & 5,7 \\
Vitaminas & 4 & 4,5 \\
Orexígenos & 4 & 4,5 \\
Outros & 9 & 10,2 \\
Total & 88 & 100,0 \\
\hline
\end{tabular}

crianças (12,3\%) apresentaram déficit de altura/idade, enquanto o déficit peso/idade foi verificado em 37 crianças $(9,3 \%)$.

A maioria das crianças $(57,0 \%)$ pertencia a famílias cuja renda familiar mensal não ultrapassava um salário mínimo e 59,3\% compartilhavam o domicílio com outras crianças menores de cinco anos de idade. Do total, $13 \%$ das mães nunca foram à escola, $23 \%$ estudaram por oito ou mais anos e $71,4 \%$ tinham no máximo 24 anos de idade (Tabela 3).

A renda familiar esteve associada de modo significativo ao uso de suplementos, o qual aumentou proporcionalmente à elevação da renda $(p=0,001)$, com OR de 3,26 (IC95\%: 1,56-6,8) para as famílias que recebem de dois a três salários mínimos, quando comparadas com as famílias com renda abaixo de um salário mínimo. Houve associação entre a suplementação vitamínica e/ou mineral e o número de crianças menores de cinco anos residentes no domicílio, verificando-se uma maior utilização do recurso de suplementação medicamentosa pelas famílias que tinham apenas um filho $(p=0,026)$. Observou-se que o uso de suplementos pelas crianças cresceu com a idade das mães $(p<0,001)$, sendo maior entre aquelas cujas mães tinham idades na faixa de 25 anos ou mais, com OR de 4,52(IC95\%: 1,99-10,47). A escolaridade materna não esteve associada ao uso de suplementos (Tabela 3).

A análise da situação alimentar demonstrou a dramática redução do aleitamento materno, com percentuais inferiores a $40 \%$ a partir da $17^{\mathrm{a}}$ semana

Tabela 3

Distribuição das crianças e uso de suplementos vitamínicos e/ou minerais, segundo variáveis sociais e biológicas. Zona da Mata Meridional de Pernambuco, 1993 a1994.

\begin{tabular}{|c|c|c|c|c|c|c|c|}
\hline \multirow[t]{3}{*}{ Variáveis } & \multirow[t]{3}{*}{ Total } & \multicolumn{4}{|c|}{ Uso de suplementos } & \multirow[t]{3}{*}{ OR (IC95\%) } & \multirow[t]{3}{*}{$p$} \\
\hline & & \multicolumn{2}{|c|}{ Sim } & \multicolumn{2}{|c|}{ Não } & & \\
\hline & & $\mathrm{n}$ & $\%$ & $\mathrm{n}$ & $\%$ & & \\
\hline \multicolumn{8}{|c|}{ Renda familiar (SM) } \\
\hline 1 & 229 & 32 & 14,0 & 197 & 86,0 & 1,00 & \\
\hline $1-12$ & 118 & 25 & 21,2 & 93 & 78,8 & $1,65(0,89-3,07)$ & 0,12 \\
\hline $2-13$ & 52 & 18 & 34,6 & 34 & 65,4 & $3,26(1,56-6,80)$ & $<0,001$ \\
\hline \multicolumn{8}{|c|}{$\mathrm{N}^{\circ}$. de crianças $<5$ anos no domicílio } \\
\hline 2 ou mais & 229 & 34 & 14,8 & 195 & 85,2 & 1,00 & \\
\hline 1 & 170 & 41 & 24,1 & 129 & 75,9 & $1,82(1,07-3,12)$ & 0,026 \\
\hline \multicolumn{8}{|c|}{ Escolaridade materna (anos)* } \\
\hline $0-3$ & 171 & 27 & 15,8 & 144 & 84,2 & 1,00 & \\
\hline $4-7$ & 135 & 62 & 19,3 & 109 & 80,7 & $1,27(0,67-2,40)$ & 0,52 \\
\hline 8 ou mais & 91 & 21 & 23,3 & 70 & 76,9 & $1,60 \quad(0,81-3,17)$ & 0,20 \\
\hline \multicolumn{8}{|c|}{ Idade materna (anos) } \\
\hline 19 & 141 & 20 & 14,2 & 121 & 85,8 & 1,00 & \\
\hline 20 a 24 & 144 & 24 & 16,7 & 120 & 83,3 & $1,21(0,61-2,42)$ & 0,68 \\
\hline 25 & 114 & 31 & 27,2 & 83 & 72,8 & $4,52(1,99-10,47)$ & $<0,001$ \\
\hline
\end{tabular}

*Em dois casos o informante não sabia dizer a escolaridade da mãe; SM = Salário mínimo; $p=0,001 ; p=0,02$. 
de vida e a precoce introdução da alimentação complementar, verificando-se que $47,6 \%$ e $30 \%$ das crianças já tinham iniciado, respectivamente, a ingestão de mingau e leite a partir da quarta semana de vida (Figura 1).

Na Tabela 4 verifica-se que a utilização de suplementos vitamínicos e/ou minerais diferiu segundo a mediana de duração do aleitamento materno, sendo essa de 77 dias (35-142) entre as crianças que fizeram uso de suplementação vitamínica e/ou mineral e de 97,5 dias $(52,5-182)$ entre as que não receberam suplementos $(p=0,01)$.

O uso de suplementos não esteve associado ao estado nutricional das crianças (altura/idade, $p=0,50$; peso/idade, $p=0,84)$. Da mesma forma, não diferiu segundo o peso ao nascer $(p=0,66)$, o sexo $(p=0,51)$, a assistência ao pré-natal $(p=0,21)$, ocorrência de consultas médicas $(p=0,78)$ e hospitalizações da criança $(p=0,86)$.

\section{Discussão}

A promoção efetiva da saúde e nutrição infantil passa necessariamente por procedimentos que envolvem a visão da questão alimentar em suas diferentes perspectivas: econômica, nutricional, social e cultural.

A comparação dos achados do presente estudo, com dados de outras pesquisas, torna-se limitada por dois motivos. Primeiro, pela quase inexistência em nosso meio de estudos voltados para a faixa etária de

Figura 1

Perfil alimentar das crianças. Zona da Mata Meridional de Pernambuco, 1993 a 1994

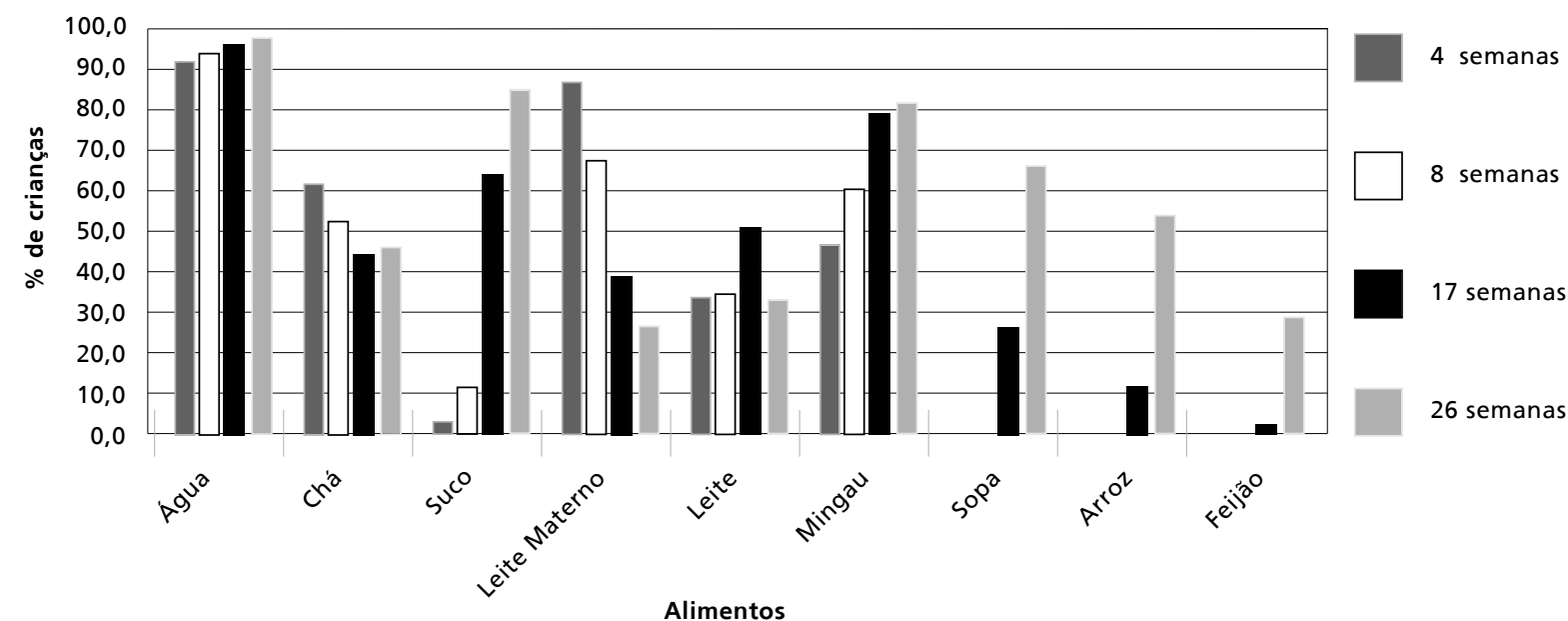

Tabela 4

Uso de suplementos vitamínicos e/ou minerais segundo a idade das crianças. Zona da Mata Meridional de Pernambuco, 1993 a 1994.

\begin{tabular}{|c|c|c|c|c|}
\hline \multirow[t]{2}{*}{ Uso de suplementos } & \multicolumn{2}{|c|}{ Duração aleitamento materno misto } & \multirow[t]{2}{*}{ Total } & \multirow[t]{2}{*}{ Teste Mann Whitney-U } \\
\hline & $\begin{array}{l}\text { Mediana } \\
\text { (dias) }\end{array}$ & $\begin{array}{c}\text { IIQ* } \\
(25-75 \%)\end{array}$ & & \\
\hline Sim & 77 & $35-142$ & 75 & $p=0,01$ \\
\hline Não & 98 & $53-182$ & 324 & \\
\hline Total & 93 & $47-182$ & 399 & \\
\hline
\end{tabular}

* Intervalo Inter-quartilar $\left(1^{\circ}=25 \%\right.$ e $\left.3^{\circ}=75 \%\right)$ 
menores de seis meses, sendo encontrado apenas um estudo, que investigou o consumo de medicamentos no primeiro trimestre de vida. ${ }^{16}$ Segundo, por tratarse de um estudo longitudinal, e por conseguinte as informações coletadas referem-se ao seguimento dos vários momentos relativos ao acompanhamento das crianças.

Ressalvadas as dificuldades referidas e objetivando situar melhor os achados deste estudo, serão estabelecidas comparações com os resultados de algumas pesquisas.

A utilização de suplementos vitamínicos e/ou minerais encontrada na $17^{\mathrm{a}}$ semana de vida foi semelhante àquela descrita por Weiderpass et al. ${ }^{16}$ para crianças no terceiro mês de vida (6\%), ao passo que na faixa etária correspondente a um mês ela foi menor, sendo de 5\% na população de Pelotas, Rio Grande do Sul, e de 1,5\% entre as crianças da Zona da Mata Meridional de Pernambuco. Para a $26^{\mathrm{a}}$ semana, correspondendo à idade de seis meses, não é possível estabelecer comparações, pela falta de dados no estudo de Pelotas, porém é notável a elevação do uso de suplementos nessa idade, o qual praticamente duplicou em relação à $17^{\mathrm{a}}$ semana de vida. No que se refere ao consumo geral, os achados do presente estudo $(18,8 \%)$ revelam níveis de consumo superiores ao encontrado por Simões e Farache 17 para menores de cinco anos $(12,9 \%)$ e por Bricks e Leone 12 para crianças menores de sete anos, situando-se também acima dos resultados encontrados por Béria et al.13 em crianças na faixa etária de três a quatro anos $(9,5 \%)$.

Em relação aos grupos farmacológicos utilizados, destacaram-se os recalcificantes, que representaram $56,8 \%$ do total de suplementos, seguidos pelos polivitamínicos $(18,8 \%)$ e os antianêmicos, que foram usados por $5,7 \%$ das crianças. Vale destacar a utilização dos estimulantes de apetite encontrada neste estudo, representando $4,5 \%$ do consumo dos produtos referidos pelas mães ou pessoa responsável como sendo "vitaminas". Béria et al.13 também ressaltaram o uso desses produtos, cuja participação no total de medicamentos consumidos chegou a $4,5 \%$.

O maior uso de suplementos pelas crianças cujas famílias possuíam melhor nível de renda, encontrado neste estudo, também foi observado por Béria et al., 13 considerando a renda per capita; por Milner et al. ${ }^{19}$, considerando a renda anual, e também por Weiderpass et al., 16 trabalhando com o conceito de classe social. Observa-se, assim, que a viabilidade econômica de adquirir o suplemento tem grande importância na decisão de oferecer esses produtos às crianças.
Por sua vez, a ausência de estudos que investiguem a influência da idade materna no uso de medicamentos por crianças não permite a comparação dos resultados encontrados no presente trabalho, onde se verificou a elevação da utilização da suplementação medicamentosa com o aumento da idade materna, especialmente entre aquelas com idade superior a 25 anos.

Observou-se que os lactentes que não compartilhavam o domicílio com outra criança menor de cinco anos estavam mais expostos ao uso de suplementos vitamínicos e/ou minerais, fato que de certa forma está relacionado com os achados descritos por Béria et al. ${ }^{13}$ para crianças na faixa etária de três a quatro anos e por Weiderpass et al.16 entre as crianças no primeiro trimestre de vida. Como assinalado por esses autores, as primíparas constituem um grupo para o qual deve ser destinada uma atenção especial para evitar o uso desnecessário de medicamentos. No caso dos suplementos vitamínicos e minerais, essa atenção deve ser voltada para corrigir as distorções no uso desses medicamentos, através de uma orientação especialmente dirigida para a fonte alimentar dessas substâncias e o seu valor para a saúde.

A ausência de associação entre a utilização de suplementos vitamínicos e minerais e o número de consultas médicas e hospitalizações difere dos resultados de outros estudos realizados, 13,16 onde o médico foi o principal responsável pela indicação de medicamentos. Outros autores também chamam a atenção para a importância desse profissional como principal responsável pela indicação de medicamentos. 1,25

O uso mais elevado de suplementos vitamínicos e/ou minerais pelas crianças cuja duração mediana do aleitamento materno foi menor condiz com os achados de Weiderpass et al. 16 sobre o maior consumo de medicamentos entre as crianças não amamentadas exclusivamente e as desmamadas, reforçando a sugestão desse autor no sentido da realização de pesquisas destinadas a explorar melhor a associação entre o aleitamento materno e uma maior utilização de medicamentos.

A precocidade de introdução de alimentos complementares e/ou substitutivos do leite materno, desde a quarta semana de vida, pode se constituir em uma prática que, se não forem observados os procedimentos corretos no preparo e administração dos alimentos, representará um importante fator de risco à saúde, devido à imaturidade fisiológica do sistema digestivo nos primeiros meses de vida, assim como pela possibilidade de contaminação microbiológica dos alimentos. 22,23 
As razões para o uso de suplementos vitamínicos e/ou minerais, assim como para o aumento no consumo verificado após a $17^{\mathrm{a}}$ semana de vida, não puderam ser investigadas neste estudo devido às limitações citadas anteriormente. Talvez os motivos que levaram ao consumo digam respeito às considerações feitas por Cordeiro quando afirma que

"[...] o uso difundido das vitaminas deve estar associado às precárias condições de alimentação e às representações difundidas pelo saber médico e por práticas ideológicas dominantes quanto ao valor das vitaminas para uma vida saudável." (1980: 186). 24

Essa difusão conduz a uma hipervalorização dos produtos farmacêuticos como via de ingestão de vitaminas, seja pela dificuldade de acesso à fonte alimentar ou pela falta de compreensão das necessidades nutricionais, podendo ser utilizados como substitutos de outras categorias de nutrientes. 1

Apesar das dificuldades na comparação dos resultados devido à escassez de dados na literatura, foi possível verificar, neste estudo, a existência de um uso de suplementos vitamínicos e minerais associado à renda familiar, ao número de crianças menores de cinco anos no domicílio e à idade materna, delineando-se, assim, uma situação onde o consumo de suplementos parece ter sido devido não às condições carenciais das crianças ou a qualquer doença que justificasse o seu uso, mas, sim, a outros fatores ligados à influência sinérgica do modelo biomédico reducionista e medicalizador e do modelo produtivo. Por sua vez, a associação do consumo de suplementos com a redução da duração do aleitamento materno provavelmente reflete uma tentativa de compensar as dificuldades experimentadas na lactação e que levam à introdução precoce da alimentação complementar.

Embora não tendo a informação sobre o comprometimento do orçamento familiar com a aquisição dos suplementos, uma vez que o banco original não tinha o objetivo de estudar em profundidade a questão do uso de medicamentos, é sabido que

\section{Referências}

1. Barros JAC. A propaganda de medicamentos. Atentado à saúde? São Paulo: Hucitec; 1995.

2. Brasil. Câmara dos Deputados. Relatório da Comissão Parlamentar de Inquérito - Medicamentos. Brasília; 2000. Relator: Dep. Ney Lopes. Presidente Dep. Nelson Marchezan.

3. Nascimento MC, Martinez DS, Luz MT. O medicamento na visão da imprensa escrita. Rio de Janeiro: Instituto de nessas áreas o suporte medicamentoso através da rede pública de saúde é insuficiente e esporádico, especialmente em se tratando dos medicamentos que foram utilizados pelas crianças estudadas. Percebese, assim, em uma população pobre, cuja renda familiar mais alta não ultrapassava três salários mínimos mensais, e que provavelmente não dispunha de recursos suficientes para alimentar adequadamente seus filhos, um gasto desnecessário com medicamentos cujas razões para o uso estão relacionadas à própria situação carencial em que vivem. Essa procura por soluções medicamentosas pode ser traduzida pela busca da saúde como uma mercadoria, pela solução mágica para problemas determinados socialmente.

Algumas medidas direcionadas à promoção de ações que visem à ampliação da consciência sanitária da população poderiam ser instrumentos de mudanças de práticas e condutas. Por meio da formação de grupos focais com gestantes, poderão ser geradas oportunidades de discussão nas quais suas crenças e valores sobre alimentação, medicamentos, cuidados com a saúde, comportamento normal do recém-nascido sejam colocados em evidência, diminuindo a ansiedade das mães no cuidado com a saúde da criança e propiciando a mudança de comportamentos não desejáveis.

Recomenda-se ainda que as instituições formadoras de recursos humanos em saúde promovam a utilização racional de medicamentos em crianças, acentuando os danos à saúde e econômico-sociais do seu uso abusivo.

Diante da condição pioneira deste estudo na pretensão de desvendar as relações existentes entre o uso de suplementos vitamínicos e a conduta alimentar em crianças tão jovens, os achados apontam para a necessidade de investigações mais abrangentes, que possam esclarecer algumas questões aqui colocadas e conduzir a ações e intervenções efetivas.
Medicina da Universidade do Estado do Rio de Janeiro; 1998. (Série Estudos em Saúde Coletiva, 174).

4. Chetley A. Las vitaminas: las vitaminas estimulan el crescimiento ... de la industria farmacêutica. In: Chetley A. Medicamentos problema.Chimbote (Peru): Acción Internacional por la Salud en América Latina y El Caribe; 1994. p. 249-62.

5. Batista Filho M. Alimentação nutrição \& saúde. In: 
Rouquayrol MZ, Almeida Filho N. Epidemiologia \& saúde. Rio de Janeiro: Medsi; 1999. p. 353-74.

6. Leão E, Starling ALP. Comentário sobre o uso de vitaminas. Rev Med Minas Gerais 1999; 9: 70-1.

7. Council on Scientific Affaire. Vitamin preparations as dietary supplements and as therapeutic agents. JAMA 1987; 257: 1929-36.

8. Dewey KG, Domellöf M, Cohen RJ, Rivera LL, Hernell O, Lönnerdal B. Iron supplementation affects grow and morbidity of breast-fed infants; results of a randomized trial in Sweden and Honduras. J Nutr 2002; 132: 3249-55.

9. Bahl R, Bhandari N, Wahed MA, Kumar WGT, Bhan MK. WHO/CHD imunization-linked vitamina a group.Vitamin A supplementation of women postpartum and of their infants at immunization alters breast milk retinol and infant vitamin A status. J Nutr 2002; 132: 3243-8.

10. West KPJr, Katz J, Shrestha SR, LeClerq SC, Khatry SK, Pradham EK, Adhikaari R, Wu LS-F, Pokhrel RP, Sommer A. Mortality of infants $<6$ mo of age supplemented with vitamin A: a randomized, double-masked trial in Nepal. Am J Clin Nutr 1995; 62: 143-8.

11. Rivera M, Téllez-Rojo M, Rosado JL, Brown KH. Múltiple micronutrient supplementation increase the growth of Mexican infants. Am J Clin Nutr 2001; 74: 657-63.

12. Bricks LF, Leone C. Utilização de medicamentos por crianças atendidas em creches. Rev Saúde Pública 1996; 30 : 527-35.

13. Béria JU, Víctora CG, Barros FC, Teixeira AB, Lombardi C. Epidemiologia do consumo de medicamentos em crianças de centro urbano da região Sul do Brasil. Rev Saúde Pública 1993; 27: 95-104.

14. Kovar MG. Use of medications and vitamin-mineral supplements by children and youths. Public Health Rep 1985; 100: 470-3.
15. Rylance GW, Wood GC, Cullen RE, Rylance ME. Use of drugs by children. Br Med J 1988; 297: 445-7.

16. Weiderpass E, Béria JU, Barros FC, Víctora CG, Tomasi E, Halpern R. Epidemiologia do consumo de medicamentos no primeiro trimestre de vida em um centro urbano do Sul do Brasil. Rev Saúde Pública 1998; 32: 335-44.

17. Simões MJS, Farache AF. Consumo de medicamentos em região do estado de São Paulo (Brazil). Rev Saúde Pública 1988; 22: 494-9.

18. Carmo TA, Lopes FC, Alves JM. Indicadores de prescrições medicamentosas: ferramentas para intervenção. Saúde em Debate 2003; 5: 49-55.

19. Milner JD, Stein DM, McCarter R, Moon RY. Early infant multivitamin supplementation is associated with increased risk for food allergy and asthma. Pediatrics 2004; 114: 27 32.

20. Lira PIC, Asworth A, Morris SS. Low birth weigh and morbidity from diarrhea and respiratory infection in Northeast Brazil. J Pediatr 1996; 128: 497-504.

21. NCHS (National Center for Health Statistics). Grow curves for children birth - 18 years, United States. Vital Health Stat 1977; 11: 1-74.

22. Euclydes MP. Nutrição do lactente: base científica para uma alimentação adequada.Viçosa: Universidade Federal de Viçosa; 1998.

23. Woisky JR. Nutrição e dietética em pediatria. Rio de Janeiro: Atheneu; 1994.

24. Cordeiro HA. A indústria da saúde no Brasil. Rio de Janeiro: Graal; 1980.

25. Barros MBA. Saúde e classe social: um estudo sobre morbidade e consumo de medicamentos [tese doutorado]. Ribeirão Preto: Faculdade de Medicina de Ribeirão Preto da Universidade de São Paulo; 1983.

Recebido em 29 de junho de 2004

Versão final em 28 de julho de 2005

Aprovado em 15 de setembro de 2005 